\title{
REFLECTION OF INTERNAL WAVES BY SAND RIPPLES
}

\section{SAMIRAN MANDAL}

Khanpara High School

Khari, 24-Parganas (South), India and

\author{
UMA BASU
}

Department of Applied Mathematics University of Calcutta, 92 A.P.C. Road Calcutta-700009, India

(Received July 19, 1992 and in revised form March 13, 1993)

\begin{abstract}
An investigation is made to study the diffraction of a train of time harmonic progressive waves propagating along the surface of separation of two superposed fluids which are laterally unbounded, the upper fluid being extended infinitely upwards, the lower fluid being of finite depth with sand ripples at the bottom. The first order correction to the velocity potential for the problem of diffraction of interface waves in the presence of bottom deformation is obtained by integral transform technique after introduction of a linear frictional term in the kinematic boundary condition at the surface of separation following Lamb (1932), and the reflection and transmission coefficients are estimated for a patch of sand ripples.
\end{abstract}

KEY WORDS AND PHRASES. Internal waves, reflection and transmission of waves, deformed bottom, sand ripples.

1991 AMS SUBJECT CLASSIFICATION CODE(S). 76B.

\section{INTRODUCTION.}

Gorgui and Kassem [1] studied generation of short internal waves produced by a cylindrical wavemaker at the surface of separation of two inviscid fluids. The studies of Gorgui and Kassem [2], Rhodes-Robinson [3] on the generation of time-harmonic waves at the surface of separation of two superposed fluids due to various types of basic singularities provided interesting results in the theory of infinitesimal wave motion at the interface of two fluids.

When a train of surface waves is propagating in a laterally unbounded ocean over an obstacle or sand ripples at the bottom, it experiences reflection and transmission, over the deformed bottom which is interesting and important to study for both coastal protection as well as for the protection to the growth of sand ripples. Miles [4], Mandal and Basu [5] estimated the reflection and transmission coefficients when a train of surface waves propagating along the surface of a single layer of fluid is incident obliquely on a cylindrical deformed bottom.

In the present paper an attempt has been made to determine the transmission and reflection coefficients for the diffracted waves along the interface of two laterally unbounded superposed immiscible fluids in which the upper fluid is extended to infinity upward while the lower fluid is of finite depth below the mean free surface with a small deformation in the form of a long cylinder in the lateral direction. By following Lamb [6], Davies [7] developed a method to solve this problem for a single layer of fluid by applying the Fourier transform technique. In the present paper, the method developed by Davies [7] has been used to solve a coupled the boundary value problem. 


\section{MATHEMATICAL FORMULATION OF THE PROBLEM.}

We now consider two incompressible, inviscid, homogeneous immiscible superposed fluids of densities $\rho_{1}, \rho_{2}\left(\rho_{1}>\rho_{2}\right)$ with the lower fluid of density $\rho_{1}$. The mean surface of separation is horizontal in equilibrium and taken as $x z$-plane, $y$-axis pointing vertically downward. The lower liquid occupying the laterally unbounded region $-\infty<x<\infty, 0 \leq y \leq h+\zeta(z)$ while the upper fluid of density $\rho_{2}$ in the region $y \leq 0,-\infty \leq x \leq \infty$.

The bottom of the ocean with small cylindrical deformation is described by $y=h+\zeta(x)$, where

$$
\left.\begin{array}{llll}
\zeta(x) & =0 & \text { for } & -\infty<x \leq L_{1} \\
=y_{b}(x) & \text { for } & L_{1} \leq x \leq L_{2} \\
=0 & \text { for } & L_{2} \leq x<\infty
\end{array}\right\}
$$

and $\zeta(x)$ together with its derivatives are assumed to be very small.

We consider the motion under gravity of the two layer of fluids when a train of progressive waves is propagating along the surface of separation of two fluids. The motion is assumed to be irrotational and simple harmonic in time so that it can be described by the velocity potential $\operatorname{Re}\{\Phi(x, y, t)\}$ for lower fluid and $\operatorname{Re}\{\Psi(x, y, t)\}$ for the upper fluid, $\Phi$ and $\Psi$ satisfy following equations and boundary conditions

$$
\begin{array}{ll}
\nabla^{2} \Phi=0, & 0 \leq y \leq h+\zeta(x), \\
\nabla^{2} \Psi=0, & y \leq 0, \\
\Phi_{y}=\Psi_{y} & \text { on } y=0, \\
\Phi_{t t}-g \Phi_{y}=s\left(\Psi_{t t}+g \Psi_{y}\right) & \text { on } y=0, \\
\Phi_{\widehat{n}}=0 & \text { on } y=h+\zeta(x), \\
\nabla \Psi \rightarrow 0 & \text { as } y \rightarrow-\infty,
\end{array}
$$

where ' $\widehat{n}$ ' denotes the inward drawn normal to the bottom, $s=\frac{\rho_{2}}{\rho_{1}}$. Let $\phi$ and $\psi$ be the effect of the bottom deformation in $\Phi$ and $\Psi$ so that

$$
\Phi=\Phi_{o}+\phi, \quad \Psi=\Psi_{o}+\psi
$$

where $\Phi_{o}, \Psi_{o}$ are prescribed first order velocity potential and $\phi, \psi$ are second order perturbed velocity potential for lower and upper fluids. The prescribed value of $\Phi_{o}$ and $\Psi_{o}$ are given by

$$
\begin{aligned}
\Phi_{o} & =\widetilde{A} \frac{\cosh \left|k_{o}\right|(h-y)}{\sinh \left|k_{o}\right| h} \cos \left(k_{o} x-n t\right) \\
\Psi_{o} & =-\widetilde{A} e^{\left|k_{o}\right| y} \cos \left(k_{o} x-n t\right)
\end{aligned}
$$

along positive $x$-direction where $k_{o}>0, \widetilde{A}$ is a known quantity depending on $n, k_{o}, g$ and $h, k_{o}$ being the unique real zero of

$$
K(\cosh |k| h+s \sinh |k| h)-(1-s)|k| \sinh |k| h=0
$$

where $n=\sqrt{K g}$ is the circular frequency.

As this wave train is normally incident upon the deformation, it experiences reflection and 
transmission over the deformation and the motion is governed by the set of equations (2.2) to (2.7), then $\phi$ and $\psi$ satisfy set of equations

$$
\begin{array}{ll}
\nabla^{2} \phi=0 & \text { in } 0 \leq y \leq h, \\
\nabla^{2} \psi=0 & \text { in }-\infty<y \leq 0, \\
\phi_{y}=\psi_{y} & \text { on } y=0, \\
s\left(\psi_{t t}-g \psi_{y}\right)=\phi_{t t}-g \phi_{y} & \text { on } y=0, \\
-\Phi_{o x} \zeta_{x}(x)+\zeta(x) \Phi_{o y y}+\phi_{y}=0 & \text { on } y=h,
\end{array}
$$

where $\Phi_{o}$ and $\zeta(x)$ is given in (2.9) and (2.1).

By the use of (2.1) in (2.16), the bottom boundary condition leads to the following relations

$$
\left.\begin{array}{llc}
\phi_{y}=0 & \text { for } & -\infty<x \leq L_{1} \\
=-U(x, t) & \text { for } & L_{1} \leq x \leq L_{2} \\
=0 & \text { for } & L_{2} \leq x<\infty
\end{array}\right\} \text { at } y=h
$$

where

$$
U(x, t)=-\zeta(x) \Phi_{\text {oyy }}(x, h, t)+\zeta_{x}(x) \Phi_{o x}(x, h, t) .
$$

We obtain a solution which corresponds to outgoing waves in the limit when it exists i.e., the asymptotic behavior of $\phi(x, y, t)$ and $\psi(x, y, t)$ as $|x| \rightarrow \pm \infty$.

\section{METHOD OF SOLUTION.}

To solve the boundary value problem (2.12) to (2.16) we use Fourier transform defined by

$$
\begin{gathered}
\widehat{f}(\xi, y, t)=\frac{1}{\sqrt{2 \pi}} \int_{-\infty}^{\infty} f(x, y, t) e^{\imath \xi x} d x \\
f(x, y, t)=\frac{1}{\sqrt{2 \pi}} \int_{-\infty}^{\infty} \widehat{f}(\xi, y, t) e^{-\imath \xi x} d \xi .
\end{gathered}
$$

Following Lamb [6], we introduce a linear frictional term in (2.15) to obtain

$$
s\left[\psi_{t t}-g \psi_{y}\right]=\phi_{t t}-g \phi_{y}+\mu \phi
$$

where $\mu$ is very small frictional parameter.

Then application of the Fourier transform to the set of equations (2.12) to (2.14), (2.16) and (3.3) gives

$$
\begin{array}{ll}
\widehat{\phi}_{y y}-\xi^{2} \widehat{\phi}=0 & \text { in } 0 \leq y \leq h, \\
\widehat{\psi}_{y y}-\xi^{2} \widehat{\psi}=0 & \text { in } y \leq 0, \\
\widehat{\phi}_{y}=\widehat{\psi}_{y} & \text { on } y=0, \\
s\left[\widehat{\psi}_{t t}-g \widehat{\psi}_{y}\right]=\widehat{\phi}_{t t}-g \widehat{\phi}_{y}+\mu \widehat{\phi}_{t} & \text { on } y=0, \\
\widehat{\phi}_{y}(\xi, t)=\frac{1}{\sqrt{2 \pi}} \int_{-\infty}^{\infty} U(x, t) e^{i \xi x} d x=\frac{T(\xi, t)}{\sqrt{2 \pi}} & \text { on } y=h .
\end{array}
$$

Equations (3.4) and (3.5) possess solution given by

$$
\widehat{\phi}=A(\xi, t) \cosh |\xi| y+B(\xi, t) \sinh |\xi| y,
$$




$$
\widehat{\psi}=b(\xi, t) e^{|\xi| y} .
$$

Boundary conditions (3.6) and (3.7) give the relation among $A(\xi, t), B(\xi, t), T(\xi, t)$ as

$$
\begin{gathered}
A(\xi, t)|\xi| \sinh |\xi| h+B(\xi, t)|\xi| \sinh |\xi| h=\frac{T}{\sqrt{2 \pi}} \\
A_{t t}(\xi, t)+\mu A_{t}(\xi, t)-s B_{t t}(\xi, t)-g|\xi|(1-s) B(\xi, t)=0 .
\end{gathered}
$$

We set

$$
\left(\begin{array}{l}
A \\
B \\
T
\end{array}\right)=\left(\begin{array}{ll}
A_{1} & A_{2} \\
B_{1} & B_{2} \\
T_{1} & T_{2}
\end{array}\right) \quad\left(\begin{array}{c}
\cos n t \\
\sin n t
\end{array}\right) .
$$

Using (3.11), (3.12) and (3.13) we have the solution given by

$$
\begin{array}{r}
\phi=\frac{1}{\sqrt{2 \pi}} \int_{-\infty}^{\infty}\left[\left(A_{1} \cosh |\xi| y+B_{1} \sinh |\xi| y\right) \cos n t\right. \\
\left.+\left(A_{2} \cosh |\xi| y+B_{2} \sinh |\xi| y\right) \sin n t\right] e^{-\cdot \xi x} d \xi \\
\psi=\frac{1}{\sqrt{2 \pi}} \int_{-\infty}^{\infty}\left[\left(B_{1} \cosh |\xi| y+\sinh |\xi| y\right) \cos n t\right. \\
\left.+B_{2}(\cosh |\xi| y+\sinh |\xi| y) \sin n t\right] e^{-i \xi x} d \xi
\end{array}
$$

where

$$
\begin{array}{cc}
A_{1}(\xi)=\frac{\ell(\xi)}{\sqrt{2 \pi} g|\xi|} \frac{\left(p T_{1}(\xi)+q T_{2}(\xi)\right)}{\left(p^{2}+q^{2}\right)}, & A_{2}(\xi)=\frac{\ell(\xi)}{\sqrt{2 \pi} g|\xi|} \frac{\left(p T_{2}(\xi)-q T_{1}(\xi)\right)}{\left(p^{2}+q^{2}\right)} \\
B_{1}(\xi)=\frac{n^{2} A_{1}(\xi)-\mu n A_{2}(\xi)}{\ell(\xi)} & B_{2}(\xi)=\frac{\mu n A_{1}(\xi)+n^{2} A_{2}(\xi)}{\ell(\xi)} \\
\ell(\xi)=s n^{2}-g(1-s)|\xi|, p=\frac{n^{2}}{g} \cosh |\xi| h+\frac{\ell(\xi)}{g} \sinh |\xi| h \\
q=\frac{\mu n}{g} \cosh |\xi| h .
\end{array}
$$

Now to determine $\dot{\phi}$ and $\psi$ we evaluate the integrals (3.14) and (3.15) by the method of contour integration. The singularities of (3.14) and (3.15) arise from the terms

$$
p^{2}+q^{2}=0
$$

Using the complex variable $z=\xi+i \chi$, the singularities are $z=z_{p}$, say which lie near to the position $z=z_{0}$ given by $p^{2}=0$ as $q \sim \mu$ where $\mu$ is very small. These singularities $z_{0}$ are given by

$$
(1-s) z_{0}=K\left(\operatorname{coth} z_{0} h+s\right)
$$

The equation (3.18) has zeros at $z_{0}= \pm\left|k_{0}\right|$.

In addition, there exists an infinite number of poles $\pm i\left|\chi_{0}\right|$ on the imaginary axis satisfying (3.18) as 


$$
\pm i(1-s)\left|\chi_{0}\right|=K\left(i \cot \left|\chi_{0}\right| h+s\right)
$$

Set $z_{p}=z_{0}+\alpha \mu$ where $\alpha$ can be determined by equating the terms $0(\mu)$ in the equation (3.17) as

$$
\alpha= \pm \text { in } \frac{\left(K s-(1-s) z_{0}\right)}{f\left(z_{0}\right)}
$$

where

$$
f\left(z_{0}\right)=(1-s) K g-n^{2} h K-\ell\left(z_{0}\right) h\left(-K s-(1-s) z_{0}\right) .
$$

So each of original poles at $z=z_{0}$ is replaced by two poles upon the introduction of linear frictional terms. Let the residues of integrals (3.14)(3.15) be $R_{z_{p}}^{\ell}$ and $R_{z_{p}}^{u}$ respectively related to lower and upper fluids. Hence

$$
\begin{aligned}
R_{z_{p}}^{\ell}= & \frac{1}{2 \pi} R_{z_{p}}^{*}\left[\left\{\left.\left(A_{1 *} \cosh z y+B_{2 *} \sinh z y\right)\right|_{z_{p}} \cos n t\right.\right. \\
& \left.\left.+\left.\left(A_{2 *} \cosh z y+B_{2 *} \sinh z y\right)\right|_{z_{p}} \sin n t\right\} e^{-s z_{p} x}\right] \\
R_{z_{p}}^{u}= & \frac{1}{2 \pi} R_{z_{p}}^{*}\left[\left\{\left.B_{1 *}(\cosh z y+\sinh z y)\right|_{z_{p}} \cos n t\right.\right. \\
& \left.\left.+\left.B_{2 *}(\cosh z y-\sinh z y)\right|_{z_{p}} \sin n t\right\} e^{-z_{p} x}\right]
\end{aligned}
$$

where

$$
\left.\begin{array}{ll}
A_{i *}=\frac{\ell(z)}{g z} T_{v}, \quad B_{\imath *}=\frac{n^{2}}{g z} T_{\imath} \quad i=1,2 \\
R_{z_{p}}^{*}=\frac{g \sinh z_{p} h}{H\left(z_{p}\right)} \\
\text { and } \quad H\left(z_{p}\right)=n^{2} h-g(1-s) \sinh ^{2} z_{p} h
\end{array}\right\}
$$

For calculation of asymptotic limit of $\phi(x, y, t)$ as $x \rightarrow-\infty$, the contour integration is taken in the upper half plane, and the result is obtained by summing up the residues at $z_{p_{1}}=k_{0}+i \mu t_{0}$, and $z_{p_{2}}=-k_{0}+i \mu t_{0}, t_{0}>0$. In the limit $\mu \rightarrow 0$, these terms give rise to the outgoing waves from the region of bed with deformation as $x \rightarrow-\infty$ and is given by

$$
\begin{aligned}
\left.\phi(x, y, t)\right|_{x \rightarrow-\infty} & =\left.2 \pi i\left(R_{z_{1}}^{\ell}+R_{z_{p_{2}}}^{\ell}\right)\right|_{k=0} \\
& =i W_{1}(y)\left[\left.\left(T_{1}-i T_{2}\right)\right|_{-k_{0}} e^{i\left(k_{0} x+n t\right)}-\left.\left(T_{1}+T_{2}\right)\right|_{k_{0}} e^{-z\left(k_{0} x+n t\right)}\right]
\end{aligned}
$$

where

$$
W_{1}(y)=\frac{\cosh k_{0}(y-h)}{k_{0} h+(1-s) \frac{g k_{0}}{n^{2}} \sinh ^{2} k_{0} h}
$$

Again for calculation of asymptotic limit of $\phi(x, y, t)$ as $x \rightarrow \infty$ the contour of integration is taken in the lower half plane and the result is obtained by assuming

$$
z_{p_{3}}=-k_{0}-i \mu t_{0}, \quad z_{p_{4}}=k_{0}-i \mu t_{0}, \quad\left(t_{0}>0\right)
$$

and summing up the residues at $z_{p_{3}}$ and $z_{p_{4}}$ we have

$$
\left.\phi(x, y, t)\right|_{x \rightarrow \infty}=\left.2 \pi i\left(R_{z_{p_{3}}}^{\ell}+R_{z_{p_{4}}}^{\ell}\right)\right|_{\mu=0}
$$




$$
=i W_{1}(y)\left[\left.\left(T_{1}+i T_{2}\right)\right|_{-k_{0}} e^{\imath\left(k_{0} x-n t\right)}-\left.\left(T_{1}-T_{2}\right)\right|_{k_{0}} e^{-\imath\left(k_{0} x-n t\right)}\right]
$$

where $W_{1}(y)$ is given by $(3.26)$.

For calculation of $\psi(x, y, z)$ as $x \rightarrow-\infty$, the contour is taken above the real axis and considering residues at $z_{p_{1}}$ and $z_{p_{2}}$ we get

$$
\left.\psi(x, y, t)\right|_{x \rightarrow-\infty}=+i W_{2}(y)\left[\left.\left(T_{1}+i T_{2}\right)\right|_{k_{0}} e^{-\imath\left(k_{0} x+n t\right)}+\left.\left(T_{1}-T_{2}\right)\right|_{-k_{0}} e^{\imath\left(k_{0} x+n t\right)}\right]
$$

where

$$
W_{2}(y)=\frac{e^{\left|k_{0}\right| y}}{h+(1-s) \frac{g}{n^{2}} k_{0} \sinh ^{2} k_{0} h}
$$

Similarly when $x \rightarrow \infty$, taking the contour below the real axis and summing up the residues at $z_{p_{3}}$ and $z_{p_{4}}$ we have

$$
\left.\psi(x, y, t)\right|_{x \rightarrow \infty}=-i W_{2}(y)\left[\left.\left(T_{1}+i T_{2}\right)\right|_{-k_{0}} e^{\imath\left(k_{0} x-n t\right)}+\left.\left(T_{1}-i T_{2}\right)\right|_{k_{0}} e^{-\imath\left(k_{0} x-n t\right)}\right]
$$

where $W_{2}(y)$ is given by $(3.29)$.

We are interested in finding out the reflection and transmission coefficients due to the presence of sand ripples

As

$$
\phi \sim\left\{\begin{aligned}
T \Phi_{0}(x, y, t) & \text { as } x \rightarrow \infty \\
R \Phi_{0}(-x, y, t) & \text { as } x \rightarrow-\infty
\end{aligned}\right.
$$

and

$$
\psi \sim \begin{cases}T \Psi_{0}(x, y, t) & \text { as } x \rightarrow \infty \\ R \Psi_{0}(-x, y, t) & \text { as } x \rightarrow-\infty\end{cases}
$$

where $\Phi_{0}$ and $\Psi_{0}$ are given in (2.9) and (2.10) and $\phi$ and $\psi$ as $x \rightarrow \pm \infty$ in (3.25) to (3.30).

As $x \rightarrow \infty$

$$
T=\left.\frac{\phi(x, y, t)}{\Phi_{0}(x, y, t)}\right|_{x \rightarrow \infty}=\left.\frac{\psi(x, y, t)}{\Psi_{0}(-x, y, t)}\right|_{x \rightarrow \infty}
$$

and similarly as $x \rightarrow-\infty$ we get

$$
R=\left.\frac{\phi(-x, y, t)}{\Phi_{0}(-x, y, t)}\right|_{x \rightarrow \infty}=\left.\frac{\psi(-x, y, t)}{\Psi_{0}(-x, y, t)}\right|_{x \rightarrow \infty}
$$

Hence $T$ and $R$ can be expressed in terms of $k_{0}, h, n$ from (2.9), (2.10), (3.25), (3.27), (3.28), (3.30) and in $U(x, t)$ given in (2.18).

\section{APPLICATION OF THE THEORY.}

We prescribe the bed form due to presence of sand ripples as

$$
y_{b}(x)=b \sin (x+\delta) \quad \text { in } L_{1} \leq x \leq L_{2}
$$

where $\delta$ is a constant phase angle. For continuity of bed elevation we take

$$
L_{1}=\left(-s_{1} \pi-\delta\right) / \ell, \quad L_{2}=\left(s_{2} \pi-\delta\right) / \ell
$$

where $s_{1}$ and $s_{2}$ are integers so that there are $\left(s_{1}+s_{2}\right) / 2$ ripples of wave number $\ell$ in the patch.

Then from (3.25) and (2.10) as $x \rightarrow-\infty$

$$
\phi(x, y, t)=-A^{1} W_{1}(y) \frac{\left(2 k_{0} / \ell\right)}{\left(2 k_{0} / \ell\right)^{2}-1}\left[(-1)^{s_{1}} \sin \left(k_{0} x+\sigma t-2 k_{0} L_{2}\right)\right.
$$




$$
\left.-(-1)^{s_{2}} \sin \left(k_{0} x+\sigma t-2 k_{0} L_{1}\right)\right], A^{1}=b k_{0} \widetilde{A}
$$

and as $x \rightarrow \infty$ from (3.27) and (2.9) we have

$$
\phi(x, y, t)=A^{1} W_{1}(y)(2 k / \ell)\left[(-1)^{s_{1}}-(-1)^{s_{2}}\right] \sin (k x-n t)
$$

In (4.3) and (4.4) we see that interaction of incident progressive waves (2.9) with the bed forms (4.1). If we take $\delta=0$ and $L_{1}=L_{2}$, as $x \rightarrow-\infty$

$$
\phi(x, y, t)=2 A^{1} W_{1}(y) \frac{\left(2 k_{0} / \ell\right)}{\left(2 k_{0} / \ell\right)^{2}-1}(-1)^{s_{1}} \sin \left(2 k_{0} L\right) \cos \left(k_{0} x+n t\right)
$$

and as $x \rightarrow \infty$

$$
\phi(x, y, t)=0
$$

Similar results for $\psi$ can be obtained from (2.10), (3.28) and (3.30) as $x \rightarrow \pm \infty$.

Ultimately $R$ has been calculated for integral number of ripples as

$$
R=\frac{2 b k_{0}}{h+\frac{(1-s) \sinh ^{2} k_{0} h}{K}}\left[(-1)^{s_{1}}\left(\frac{2 k_{0}}{\ell}\right) \frac{\sin \frac{2 \pi k_{0}}{\ell}}{\left(\frac{2 k_{0}}{\ell}\right)-1}\right]
$$

and $T=0$.

\section{CONCLUSION AND DISCUSSION.}

The expressions (3.25), (3.27) and (3.28), (3.30) represent the reflected and transmitted velocity potential for lower and upper fluids respectively. By taking $s=0$ the results obtained by Davies [7] for a single fluid can be recovered. The results (4.5) and (4.6) explain the fact that there is no disturbance in the perturbation solution on the down-wave side of the ripple patch, but there is a reflected disturbance on the up-wave side. The result (4.5) also explains that the size of the reflected wave i.e., $W_{1}(y)$ depend on $s=\rho_{1} / \rho_{2}$ the density ratio of two fluids. The result (4.7), (4.8) give an estimate of reflection coefficients when there are integral number of ripples on sea-bed. One can also include surface tension at the surface of separation of the fluid.

ACKNOWLEDGEMENT. This work is partially supported by SAP Centre of Advanced Study in Applied Mathematics, Calcutta University.

\section{REFERENCES}

1. GORGUI, M.A. \& KASSEM, S.E., On the generation of short internal waves by cylinders oscillating at the surface separating two infinite liquids, Math. Proc. Camb. Phil. Soc. 82 (1978), 481-494.

2. GORGUI, M.A. \& KASSEM, S.E., Basic singularities in the theory of internal waves, Q. Jl. Mech. Appl. Math. 31 (1978), 31-48.

3. RHODES-ROBINSON, P.F., On waves at an interface between two liquids, Math. Proc. Camb. Phil. 88 (1980), 183-191.

4. MILES, J.W., Oblique surface-wave diffraction by a cylindrical obstacle, Dyn. Atmos. Oceans 6 (1981), 121-131.

5. MANDAL, B.N. \& BASU, U., A note on oblique water wave diffraction by cylindrical deformation on the bottom in the presence of surface tension, Arch. Mech. 42 (1990), 723-727.

6. LAMB, H., Hydrodynamics, Cambridge University Press, 6th Edition, 1932.

7. DAVIES, A.G., The reflection of wave energy by undulation on the sea bed, Dyn. Atmos. Oceans 6 (1982), 207-232. 


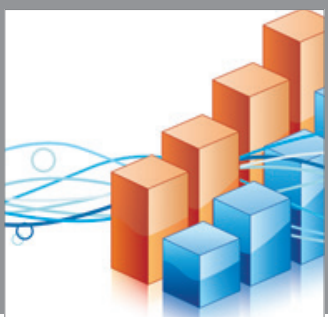

Advances in

Operations Research

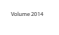

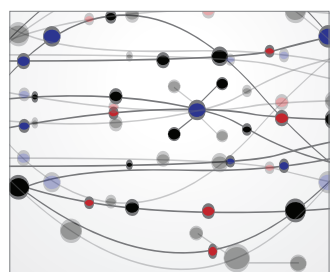

\section{The Scientific} World Journal
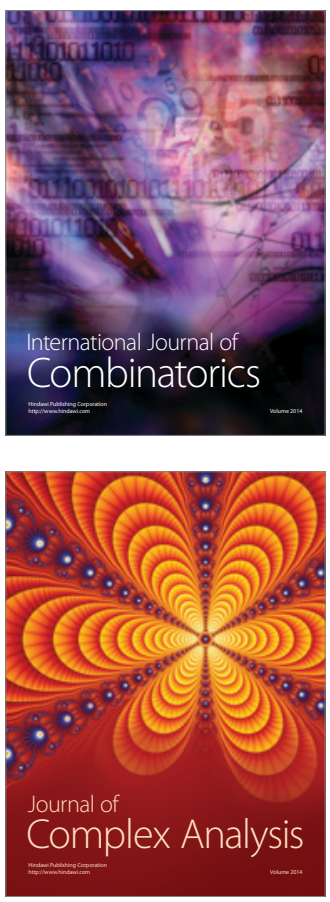

International Journal of

Mathematics and

Mathematical

Sciences
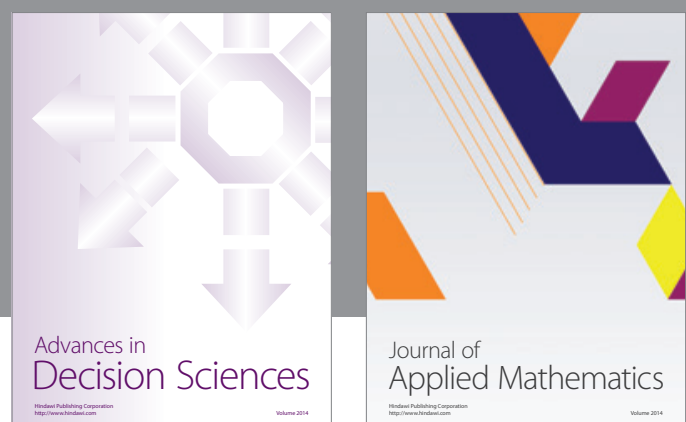

Journal of

Applied Mathematics
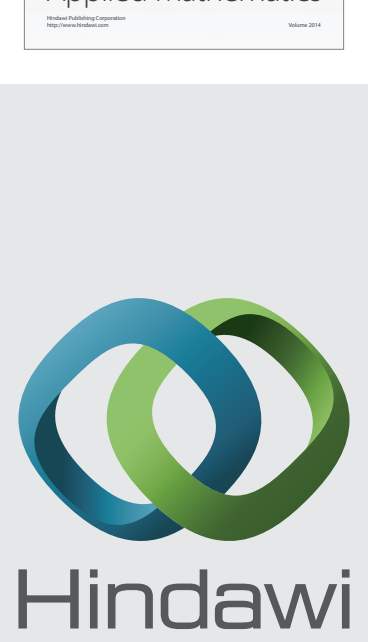

Submit your manuscripts at http://www.hindawi.com
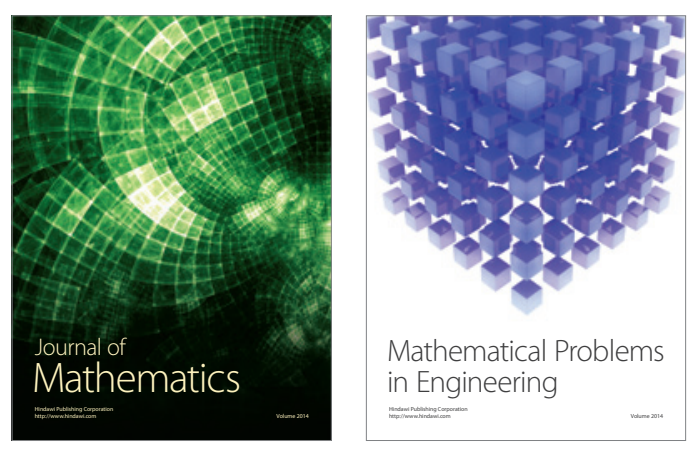

Mathematical Problems in Engineering
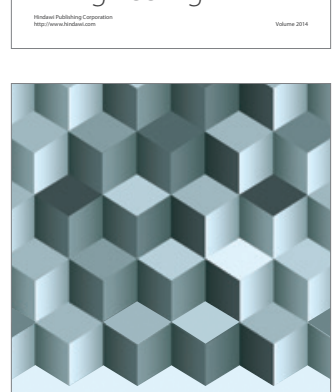

Journal of

Function Spaces
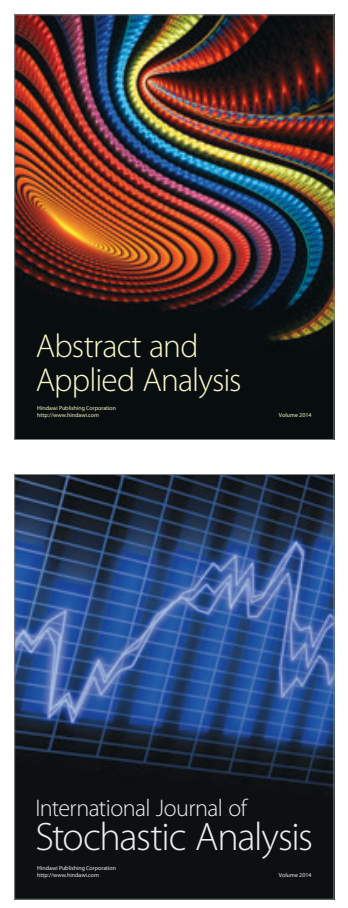

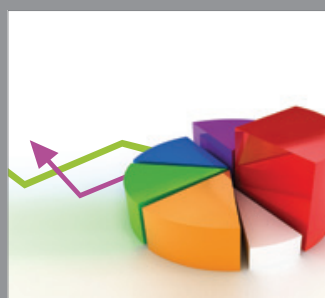

ournal of

Probability and Statistics

Promensencen
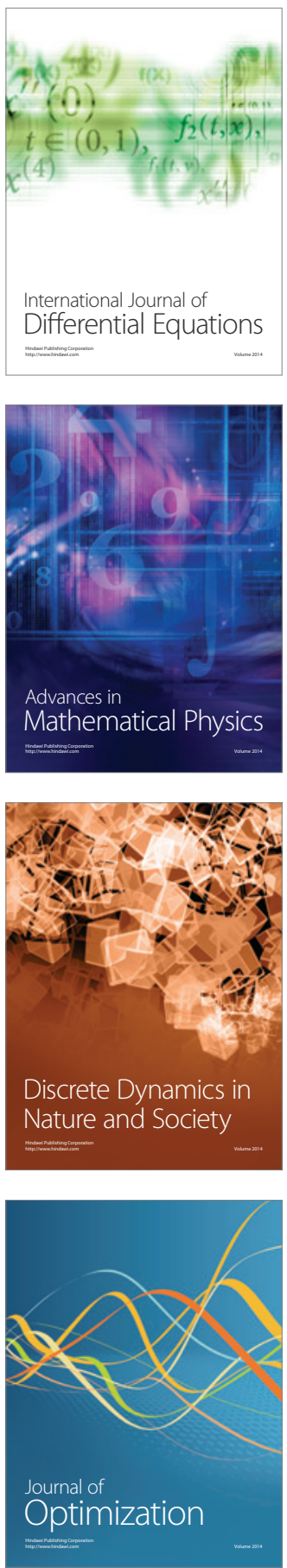